\title{
Amberlite IR-120 Catalyzed Green and Efficient One-Pot Synthesis of Benzylpyrazolyl Coumarin in Aqueous Medium
}

\author{
Ashishkumar P. Katariya ${ }^{1}$, Satish U. Deshmukh ${ }^{1}$, Sunil U. Tekale ${ }^{1}$, Maya V. Katariya ${ }^{1,}$, Rajendra P. \\ Pawar 1,* \\ 1 Department of Chemistry, Deogiri College, Aurangabad 431 005, Maharashtra, India \\ * Correspondence: rppawar@yahoo.com;
}

Scopus Author ID 7003738785

Received: 6.12.2020; Revised: 6.01.2021; Accepted: 12.01.2021; Published: 19.01.2021

\begin{abstract}
An efficient, green, and cost-effective synthesis of benzylpyrazolyl coumarin by one-pot four-component condensation of hydrazine hydrate or phenyl hydrazine, ethyl acetoacetate, aromatic aldehyde, and 4-hydroxycoumarin in the presence of Amberlite IR-120 as a catalyst in an aqueous medium has been reported. Shorter reaction time, operation simplicity, low cost of catalyst, and aqueous medium are key advantages of this method for synthesizing benzylpyrazolyl coumarin in moderate to high yield.
\end{abstract}

Keywords: benzylpyrazolyl coumarin; Amberlite IR-120; cost-effective; aqueous medium.

(C) 2020 by the authors. This article is an open-access article distributed under the terms and conditions of the Creative Commons Attribution (CC BY) license (https://creativecommons.org/licenses/by/4.0/).

\section{Introduction}

In the era of Science and Technology, the development of new routes for the synthesis of bioactive heterocyclic compounds that minimize pollution and loss of the environment has become a challenging task in organic synthesis. Green Chemistry is emerging as a powerful technique that avoids the generation and use of hazardous material and introduces powerful synthetic methods. It includes the synthesis of heterocyclic compounds with more economically and environmentally benign approaches [1-2].

Multicomponent reaction (MCR) is one of the finest techniques in organic synthesis. Three or more reactants are united together in a single vessel to form a complex heterocyclic molecule without isolating intermediate. These reactions minimize the cost and time of the reaction [3]. The use of water as a reaction medium is another green technique in organic synthesis. Water is an environmentally benign solvent that is readily available, non-toxic, inexpensive, and highly polar solvent [4]. Heterogeneous catalysis also contributes to the principle of green chemistry. It has several advantages such as reusability, operational simplicity, non-toxicity, and ease of separation from the reaction [5]. In this context, aqua mediated heterogeneously catalyzed multicomponent reactions have emerged as a powerful strategy that obeys most of Green Chemistry principles.

3-Substituted coumarin, particularly 3-benzyl substituted 4-hydroxy coumarin is an important class of oxygen-containing heterocycles present in many natural and synthetic pharmaceutical moieties, Mainly it is included in compounds like warfarin, coumatetralyl, 
phenprocoumon (Fig 1 a) and exhibit antithrombic [6], anti-bacterial, anti-HIV [7], antitumor [8] and anti-viral activities [9].<smiles>CC(=O)CC(c1ccccc1)c1c(O)c2ccccc2oc1=O</smiles>

Wafarin<smiles>CCC(c1ccccc1)c1c(O)c2ccccc2oc1=O</smiles>

Phenprocoumon<smiles>O=c1oc2ccccc2c(O)c1C1CCCc2ccccc21</smiles>

Coumatetralyl

Figure 1 a. Some bioactive 3- substituted 4- hydroxy coumarins.<smiles>CC1=NN(c2ccccc2)C(=O)C1</smiles>

Edaravone<smiles>Cc1cc(=O)n(-c2ccccc2)n1C</smiles>

Phenazone<smiles>Cc1c(N)c(=O)n(-c2ccccc2)n1C</smiles>

Ampyrone<smiles>Cc1c(C(C)C)c(=O)n(-c2ccccc2)n1C</smiles>

Propylphenazone

Figure 1 b. Some bioactive pyrazolones.

Similarly, pyrazolone is an important class of nitrogen-containing heterocycles, which is a structural unit of analgesic and antipyretic drugs [10] like phenazone, propylphenazone, and ampyrone, also a part of edaravone (Fig 1 b), which is used in treating myocardial ischemia [11] and brain disease [12]. In addition to these, pyrazolone possesses a very broad spectrum of biological activities such as anti-tubercular [13], anti-inflammatory [14], anti-depressant [15], and stimulators in gastric secretion [16].

Considering the broad range of biological activities of 3-benzyl substituted coumarin and pyrazolone as a new hybrid molecule, benzylpyrazolyl coumarin was first synthesized by P. P. Ghosh and his co-worker [17] by combining pyrazolone and 3-benzyl substituted coumarin in search of an advanced bio-active scaffold. Its synthesis was carried in an aqueous medium using glacial acetic acid as a catalyst. There are some reports which discuss the synthesis of benzyl pyrazolyl coumarin, which includes synthesis using $\mathrm{ZnO}$ as the catalyst at $120^{\circ} \mathrm{C}$ under solvent-free condition [18], $\mathrm{ZrO}_{2}$ nanoparticle catalyzed synthesis [19], [bmIm] Br ionic liquid catalyzed and mediated synthesis [20], alum catalyzed microwave synthesis in PEG [21], $\mathrm{Ca}(\mathrm{OTf})_{2}$ catalyzed synthesis in aqueous medium under reflux condition [22], Mohr's salt hexahydrate catalyzed synthesis in aqueous medium at $80^{\circ} \mathrm{C}$ [23], solvent-free synthesis catalyzed by $\mathrm{FeCl}_{3} . \mathrm{SiO}_{2}$ nanoparticles [24], melamine-modified chitosan catalyzed synthesis in ethanol: water [25] at $80^{\circ} \mathrm{C}$, multiwalled carbon nanotube (MWCNT) catalyst [26], solventfree synthesis using Nb-Zr/KIT-6 catalyst [27], and taurine catalyzed synthesis [28].

Attributable to the fact that low cost, reusability, operational simplicity, and ease in isolation after completion of reaction has made Amberlite IR-120 resin an important heterogeneous catalyst in organic synthesis [29]. In continuation of our previous work [30-32] in heterogeneous catalysis, herein we report a simple, efficient, and one-pot synthesis of benzylpyrazolyl coumarin in an aqueous medium using Amberlite IR-120 resin as a catalyst (Scheme 1). 


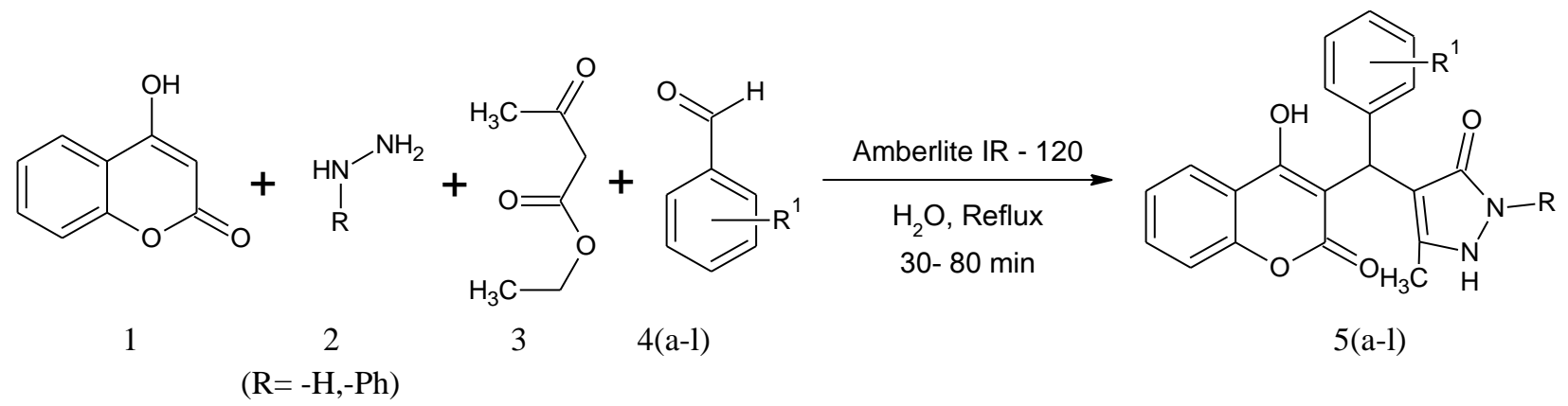

Scheme 1. General Scheme for the synthesis of benzlypyrazolyl Coumarin 5(a-m).

\section{Materials and Methods}

\subsection{Experimental.}

4-hydroxycoumarin, hydrazine hydrate, phenylhydrazine, and ethyl acetoacetate were purchased from SD fine chemical company. Most of the aldehydes were purchased from Sigma-Aldrich, and some of the aldehydes were from SD fine chemical company. All solvents used were purchased from Loba chemicals. Double distilled water was used as a reaction solvent, and catalyst Amberlite IR-120 was purchased from Loba chemical company. All the glassware used to carry out the reaction was washed with acetone and refluxed with methanol, later on, dried in an air oven at $60^{\circ} \mathrm{C}$ for 1 hour. Progress of the reaction was monitored by using TLC (Thin Layer Chromatography). All the melting points were recorded by using digital melting point apparatus. IR Spectra is recorded on FT-IR Spectrometer in $\mathrm{KBr} .{ }^{1} \mathrm{H}$ and ${ }^{13} \mathrm{C}$ NMR were recorded on Bruker (Advance Neo $500 \mathrm{MHz}$ NMR Spectrometer) at SAIF P. U. using TMS (Tetramethylsilane) as internal Standard and DMSO-d 6 as a solvent. Chemical shift values are recorded in $\delta \mathrm{ppm}$. Mass spectrometric data were recorded on waters Q-tof- micro mass at SAIF P. U. Chandigarh.

\subsection{General procedure for the synthesis of benylpyrazolyl coumarin.}

A clean $50 \mathrm{~mL}$, round bottom flask equipped with a reflux condenser was charged with hydrazine hydrate or phenyl hydrazine $(1 \mathrm{mmol})$. Ethyl acetoacetate $(1 \mathrm{mmol})$ in $10 \mathrm{~mL}$ water and the contents were heated. Amberlite IR-120 (75 mg), aromatic aldehyde (1 mmol), and 4-hydroxycoumarin were added at room temperature under stirring, and flushed reaction mass with $5 \mathrm{~mL}$ water then raised reaction mass to reflux temperature. TLC monitored maintained temperature and progress of the reaction. After completing the reaction, as monitored by TLC, the resulting reaction mass cooled to room temperature, filtered the reaction mass, and washed with $5 \mathrm{~mL}$ of water. The crude product obtained was purified by crystallization using ethanol to yield a pure product. The catalyst was removed during hot filtration in crystallization.

\subsection{Spectral data of selected compounds.}

\subsubsection{Compound (5a).}

White Solid, Yield - 92\%, IR (KBr) cm $\mathrm{cm}^{-1}$ 3134, 3080, 1647, 1622,1588,1516, 1477 , 1417, 1340, 1207, 1157, 1083, 754. ${ }^{1 \mathrm{H}} \mathrm{NMR} 500 \mathrm{MHz}$ (DMSO-d6) $\delta_{\mathrm{H}:} 2.39$ (s, 1H), 6.12 (s, $3 \mathrm{H}), 6.75-6.77(\mathrm{~m}, 1 \mathrm{H}), 6.90(\mathrm{dd}, 1 \mathrm{H}), 7.28-7.37(\mathrm{~m}, 3 \mathrm{H}), 7.50-7.53(\mathrm{~m}, 2 \mathrm{H}), 7.57-7.60(\mathrm{~m}$, 1H), 7.65-7.69 (m, 3H), 7.78-7.80 (m, 1H), 10.59 (bs, 1H), $12.92(\mathrm{~s}, 1 \mathrm{H}), 14.38(\mathrm{~s}, 1 \mathrm{H}) .{ }^{13} \mathrm{C}$ NMR $125 \mathrm{MHz}$ (DMSO-d6) $\delta_{\mathrm{C}}: 10.45,31.60,103.65,106.06,113.44,115.81,116.53,117.63$, 
$120.75,123.72,123.79,126.64,127.57,128.85,129.17,131.90,135.00,136.09$, $\left.141.10,146.81,151.79,160.81,161.88,163.11,163.19 \mathrm{LCMS}^{\mathrm{M}}+\mathrm{H}\right]^{+}=486.3045$

\subsubsection{Compound (5c).}

White Solid, Yield - 91\%, IR (KBr) $\mathrm{cm}^{-1}-3421,3066,1653,1608,1566,1417,1188$, 1041, 758., ${ }^{1} \mathrm{H}$ NMR $500 \mathrm{MHz}$ (DMSO-d6) $\delta_{\mathrm{H}:} 2.36$ (s, 3H), 5.66 (s, 1H), 7.055-7.09 (m, 2H), 7.19-7.22 (m, 2H), 7.28-7.37 (m, 3H), 7.51(t, 2H), 7.57-7.59 (m, 1H), 7.69-7.70 (m, 2H), 7.79$7.81(\mathrm{~m}, 1 \mathrm{H}), 12.9$ (bs 1H), 15.19(bs 1H). ${ }^{13} \mathrm{C}$ NMR $125 \mathrm{MHz}$ (DMSO-d6) $\delta_{\mathrm{C}}: 10.41,33.04$, $105.01,106.49,114.61,114.73,115.77,120.72,123.70,123.73,126.53,128.56,128.63$, $129.13,131.83,146.74,151.91,164.09[\mathrm{M}+\mathrm{H}]^{+}=443.3746$

\subsubsection{Compound (5d).}

White Solid, Yield - 87\%, IR (KBr) cm $\mathrm{cm}^{-1}$ 3527, 3064, 2935, 1658, 1614, 1588, 1496, 1417, 1170, 1109, 759., ${ }^{1} \mathrm{H}$ NMR $500 \mathrm{MHz}$ (DMSO-d6) $\delta_{\mathrm{H}}: 2.36$ (s, 3H), 5.61(s, 1H), 6.636.67(m, 2H), 6.95-6.97 (m, 2H), 7.29-7.37(m, 3H), 7.50-7.53 (m, 2H), 7.57-7.61 (m, 1H), 7.68$7.70(\mathrm{~m}, 2 \mathrm{H}), 7.80-7.82(\mathrm{~m}, 1 \mathrm{H}), 12.7$ (bs 1H), 15.0(bs 1H). ${ }^{13} \mathrm{C}$ NMR $125 \mathrm{MHz}$ (DMSO-d6) $\delta_{\mathrm{C}}: 10.34,32.80,90.88,105.77,106.88,114.75,115.68,115.73,116.26,117.98,120.45$, $123.69,124.80,126.47,127.97,128.67,129.27,132.07,135.13,146.74,151.80,153.41$, $155.40,161.76,162.10,163.22,165.52[\mathrm{M}+\mathrm{H}]^{+}=441.3662$

\subsubsection{Compound (5f).}

White Solid, Yield - 90\%, IR (KBr) cm $\mathrm{cm}^{-1}$ 3495, 3153, 3064, 2935, 1658, 1606, 1562 , 1496, 1417, 1045, 759., ${ }^{1} \mathrm{H}$ NMR $500 \mathrm{MHz}$ (DMSO- $\left.d_{6}\right) \delta_{\mathrm{H}}: 2.51(\mathrm{~s}, 3 \mathrm{H}), 5.72(\mathrm{~s}, 1 \mathrm{H}), 6.45-6.7$ $(\mathrm{m}, 1 \mathrm{H}), 7.1-7.2(\mathrm{~m}, 1 \mathrm{H}), 7.21-7.36(\mathrm{~m}, 3 \mathrm{H}), 7.4(\mathrm{~m}, 1 \mathrm{H}), 7.42-7.49(\mathrm{~m}, 1 \mathrm{H}), 7.5-7.6(\mathrm{~m}, 2 \mathrm{H})$, 7.6-7.75 (m, 3H), 7.76-7.86 (m 1H), 10.1 (bs, 1H), 14.1 (bs 1H). ${ }^{13} \mathrm{C}$ NMR $125 \mathrm{MHz}$ (DMSO$d_{6)} \delta_{\mathrm{C}}: 1052,29.90,104.08,106.84,109.42,115.62,116.95,118.09,120.60,123.65,126.51$, $129.18,129.48,130.88,131.52,135.13,146.71,151.63,153.25,154.21,162.48,163.60$ $[\mathrm{M}+\mathrm{H}]^{+}=521.3040$

\subsubsection{Compound $(5 \mathrm{~g})$.}

White Solid, Yield - 65\%, IR (KBr) $\mathrm{cm}^{-1}$ - 3086, 1654, 1604, 1536, 1500, 1417, 1186 , 1043, 752., ${ }^{1} \mathrm{H}$ NMR $500 \mathrm{MHz}\left(\mathrm{DMSO}-d_{6}\right) \delta_{\mathrm{H}}: 2.36(\mathrm{~s}, 3 \mathrm{H}), 5.65(\mathrm{~s}, 1 \mathrm{H}), 7.27-7.30(\mathrm{~m}, 1 \mathrm{H})$, 7.32-7.34 (m, 2H), 7.36-7.41 (m, 1H), 7.44-7.55 (m, 3H), 7.5-7.64 (m, 2H), 7.64-7.75 (m, 2H), 7.75-7.85 (m, 1H), 15.1(bs 1H). ${ }^{13} \mathrm{C}$ NMR $125 \mathrm{MHz}$ (DMSO-d6) $\delta_{\mathrm{c}}: 11.50,32.00,115.72$, $120.81,123.72,126.71,129.08,131.61,146.38,152.00[\mathrm{M}+\mathrm{H}]^{+}=493.2950$

\subsubsection{Compound (5j).}

White Solid, Yield - 70\%, IR (KBr) cm $\mathrm{cm}^{-1}$ 3321, 3124, 1647, 1608, 1508, 1456, 1234, 1176, 1043, 756; ${ }^{1} \mathrm{H}$ NMR $500 \mathrm{MHz}$ (DMSO-d6) $\delta: 2.25$ (s, 3H), $5.40(\mathrm{~s}, 1 \mathrm{H}), 6.5-6.66(\mathrm{~m}$, $2 \mathrm{H}), 6.8-7.0(\mathrm{~m}, 2 \mathrm{H}), 7.16-7.35(\mathrm{~m}, 2 \mathrm{H}), 7.43-7.56(\mathrm{~m}, 2 \mathrm{H}), 7.73-7.85(\mathrm{~m}, 1 \mathrm{H}), 9.0(\mathrm{bs}, 1 \mathrm{H})$, 12.6 (bs 1H), 13.00 (bs 1H), 17.00 (bs 1H). ${ }^{13} \mathrm{C}$ NMR $125 \mathrm{MHz}$ (DMSO-d6) $\delta_{\mathrm{C}}: 9.95,32.43$, $105.00,105.89$, 114.63, 115.56, 119.40, 123.24, 123.86, 127.54, 130.74, 131.20, 151.99, $155.17,162.12,164.54[\mathrm{M}+\mathrm{H}]^{+}=365.3070$ 


\section{Results and Discussion}

Initially, we have synthesized benzylpyrazolyl coumarin derivative (5a) by a synthetic strategy reported by A. Das and his co-worker and used this compound as a reference standard for our further investigation. A reaction of phenylhydrazine hydrate, ethyl acetoacetate, p-nitro benzaldehyde, and 4-hydroxycoumarin was used as a model reaction to study different parameters such as catalyst concentration and solvent (Scheme 2).

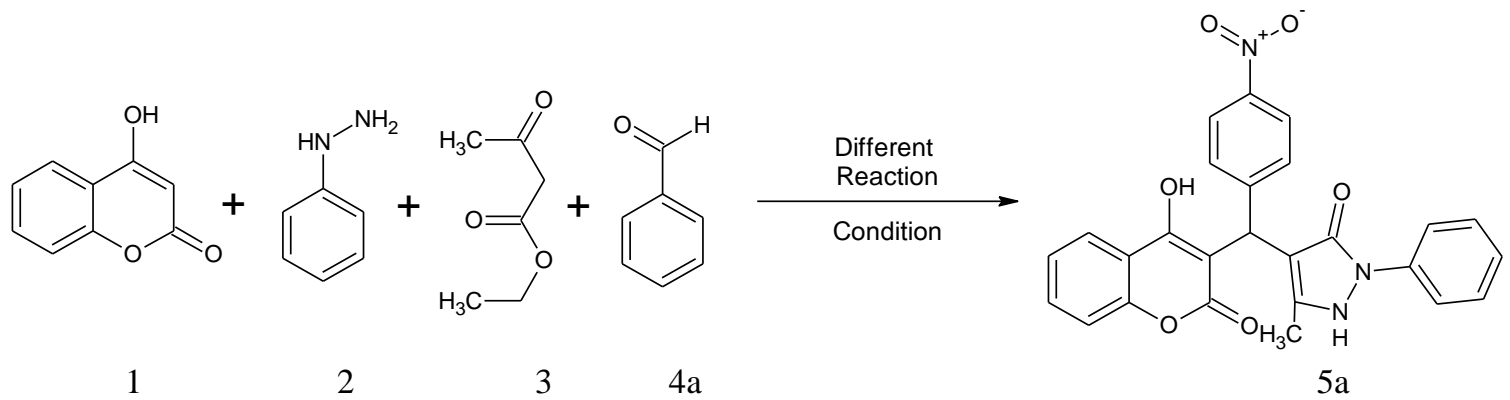

Scheme 2. Optimization of reaction conditions on the model reaction.

In the beginning, a model reaction was carried out without catalyst at room temperature to reflux condition in an aqueous medium for 9 hours. However, no product was formed, as confirmed by TLC (Table 1, entry1). Similar results are obtained by carrying out model reaction by using ethanol as a solvent under reflux condition and $100^{\circ} \mathrm{C}$ in solvent-free condition (Table 1, entries 2 and 3). In the next investigation, different catalysts such as tannic acid, ascorbic acid, and glycine were used in the model reaction using ethanol and water as solvent under reflux conditions, but very low yields were obtained. (Table 1, entries 4-10).

Table 1. Effect of different reaction conditions and catalysts on the synthesis of $5 \mathrm{a}^{\mathrm{a}}$.

\begin{tabular}{l|l|c|c|c|c} 
Entry & Catalyst & $\begin{array}{c}\text { Solvent } \\
\mathbf{( 1 5} \mathbf{~ m L})\end{array}$ & Condition & $\begin{array}{c}\text { Time } \\
(\mathbf{m i n} / \mathbf{h r})\end{array}$ & Yield $^{\mathbf{b}}(\mathbf{\%})$ \\
\hline 1 & - & Water & Reflux & $9 \mathrm{~h}$ & $-^{\mathrm{c}}$ \\
\hline 2 & - & Ethanol & Reflux & $9 \mathrm{~h}$ & $-^{\mathrm{c}}$ \\
\hline 3 & - & $\begin{array}{c}\text { Solvent- } \\
\text { free }\end{array}$ & $100^{\circ} \mathrm{C}$ & $9 \mathrm{~h}$ & $-^{\mathrm{c}}$ \\
\hline 4 & Ascorbic Acid (10 mol\%) & Water & Reflux & $9 \mathrm{~h}$ & 23 \\
\hline 5 & Ascorbic Acid (10 mol\%) & Ethanol & Reflux & $9 \mathrm{~h}$ & 14 \\
\hline 6 & Tannic Acid (10 mol\%) & Water & Reflux & $9 \mathrm{~h}$ & 28 \\
\hline 7 & Tannic Acid (10 mol\%) & Ethanol & Reflux & $9 \mathrm{~h}$ & 32 \\
\hline 8 & Glycine (10 mol\%) & Water & Reflux & $9 \mathrm{~h}$ & $-^{\mathrm{c}}$ \\
\hline 10 & Glycine (10 mol\%) & Ethanol & Reflux & $9 \mathrm{~h}$ & 12 \\
\hline $\mathbf{1 1}$ & Amberlite IR-120 (50mg) & Ethanol & Reflux & $75 \mathrm{~min}$ & 45 \\
\hline
\end{tabular}

a All reactions were carried out by phenyl hydrazine $(1 \mathrm{mmol})$, ethyl acetoacetate $(1 \mathrm{mmol})$ 4-nitro benzaldehyde $(1 \mathrm{mmol}) \&$ 4-hydroxycoumarin $(1 \mathrm{mmol}) .{ }^{\mathrm{b}}$ isolated yield of product. ${ }^{\mathrm{c}}$ Reaction failed to provide any product.

When a catalytic amount of amberlite IR-120 resin was added to the same water and ethanol reaction as a solvent, a considerable yield was obtained (Table 1, entry10, and 11). With these results, we have optimized the protic and aprotic solvents for the same reaction and found that polar protic solvent such as water, ethanol, and methanol are good solvents for reaction instead of polar aprotic solvents such as acetonitrile, toluene, dichloromethane, and carbon tetrachloride but best solvent for this reaction is water (Table 2, entries 2-5).

Table 2. Effect of different solvents on the synthesis of $5 \mathrm{a}^{\mathrm{a}}$. 


\begin{tabular}{l|l|c|c|c|c} 
Entry & Solvent $(\mathbf{1 5} \mathbf{~ m L})$ & Catalyst & Condition & $\begin{array}{c}\text { Time } \\
(\mathbf{m i n} / \mathbf{h r})\end{array}$ & $\begin{array}{c}\text { Yield }^{\mathbf{b}} \\
(\mathbf{\%})\end{array}$ \\
\hline 1 & Methanol & $\begin{array}{c}\text { Amberlite IR-120 } \\
(50 \mathrm{mg})\end{array}$ & Reflux & $90 \mathrm{~min}$ & 44 \\
\hline 2 & Acetonitrile & $\begin{array}{c}\text { Amberlite IR-120 } \\
(50 \mathrm{mg})\end{array}$ & Reflux & $9 \mathrm{~h}$ & 10 \\
\hline 3 & $\begin{array}{l}\text { Carbon tetra } \\
\text { chloride }\end{array}$ & $\begin{array}{c}\text { Amberlite IR-120 } \\
(50 \mathrm{mg})\end{array}$ & Reflux & $9 \mathrm{~h}$ & $-^{\mathrm{c}}$ \\
\hline 4 & Dichloromethane & $\begin{array}{c}\text { Amberlite IR-120 } \\
(50 \mathrm{mg})\end{array}$ & Reflux & $9 \mathrm{~h}$ & 19 \\
\hline 5 & Toluene & $\begin{array}{c}\text { Amberlite IR-120 } \\
(50 \mathrm{mg})\end{array}$ & Reflux & $9 \mathrm{~h}$ & $-^{c}$
\end{tabular}

${ }^{\text {a }}$ All reactions were carried out by phenylhydrazine $(1 \mathrm{mmol})$, ethyl acetoacetate $(1 \mathrm{mmol})$ 4-nitro benzaldehyde $(1 \mathrm{mmol}) \& 4$-hydroxycoumarin $(1 \mathrm{mmol}),{ }^{\mathrm{b}}$ Isolated yield of product, ${ }^{\mathrm{c}}$ Reaction failed to provide any product

With these delightful results obtained by using Amberlite IR-120 as the catalyst; next, we optimized the quantity of catalyst for the reaction and found that although the reaction was successful with $50 \mathrm{mg}$ of catalyst; the yield obtained was low, and the speed of the reaction was slow (Table 3, Entry11). Further increase in the quantity of catalyst to $75 \mathrm{mg}$ shows the maximum yield and reaction rate (Table 3, Entry 2). Further increase in catalyst quantity as $100 \mathrm{mg}$ and $125 \mathrm{mg}$ did not improve the reaction rate and yield of product (Table 3, Entry 4 and 5). Instead, by increasing catalyst quantity beyond $75 \mathrm{mg}$ yield of the product was decreased.

Table 3. Effect of catalyst (Amberlite IR-120) concentration on the synthesis of $5 \mathrm{a}^{\mathrm{a}}$.

\begin{tabular}{l|c|c|c|c|c} 
Entry & $\begin{array}{c}\text { Catalyst } \\
\text { concentration }(\mathbf{m g})\end{array}$ & Solvent $\mathbf{( 1 5} \mathbf{~ m L )}$ & Condition & $\begin{array}{c}\text { Time } \\
(\mathbf{m i n})\end{array}$ & Yield $^{\mathbf{b}}(\mathbf{\%})$ \\
\hline 1 & 50 & Water & Reflux & 45 & 69 \\
\hline $\mathbf{2}$ & $\mathbf{7 5}$ & Water & Reflux & $\mathbf{3 0}$ & $\mathbf{8 9}$ \\
\hline 3 & 100 & Water & Reflux & 30 & 81 \\
\hline 4 & 125 & Water & Reflux & 35 & 77
\end{tabular}

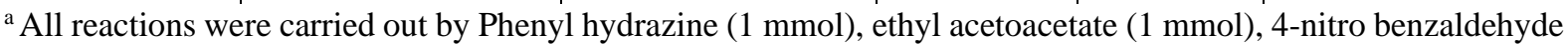
$(1 \mathrm{mmol}) \&$ 4-hydroxycoumarin $(1 \mathrm{mmol}),{ }^{\mathrm{b}}$ isolated yield of the product.

Table 4. Synthesis of benzylpyrazolyl coumarin derivatives ${ }^{\mathrm{a}}$ 5(a-1).

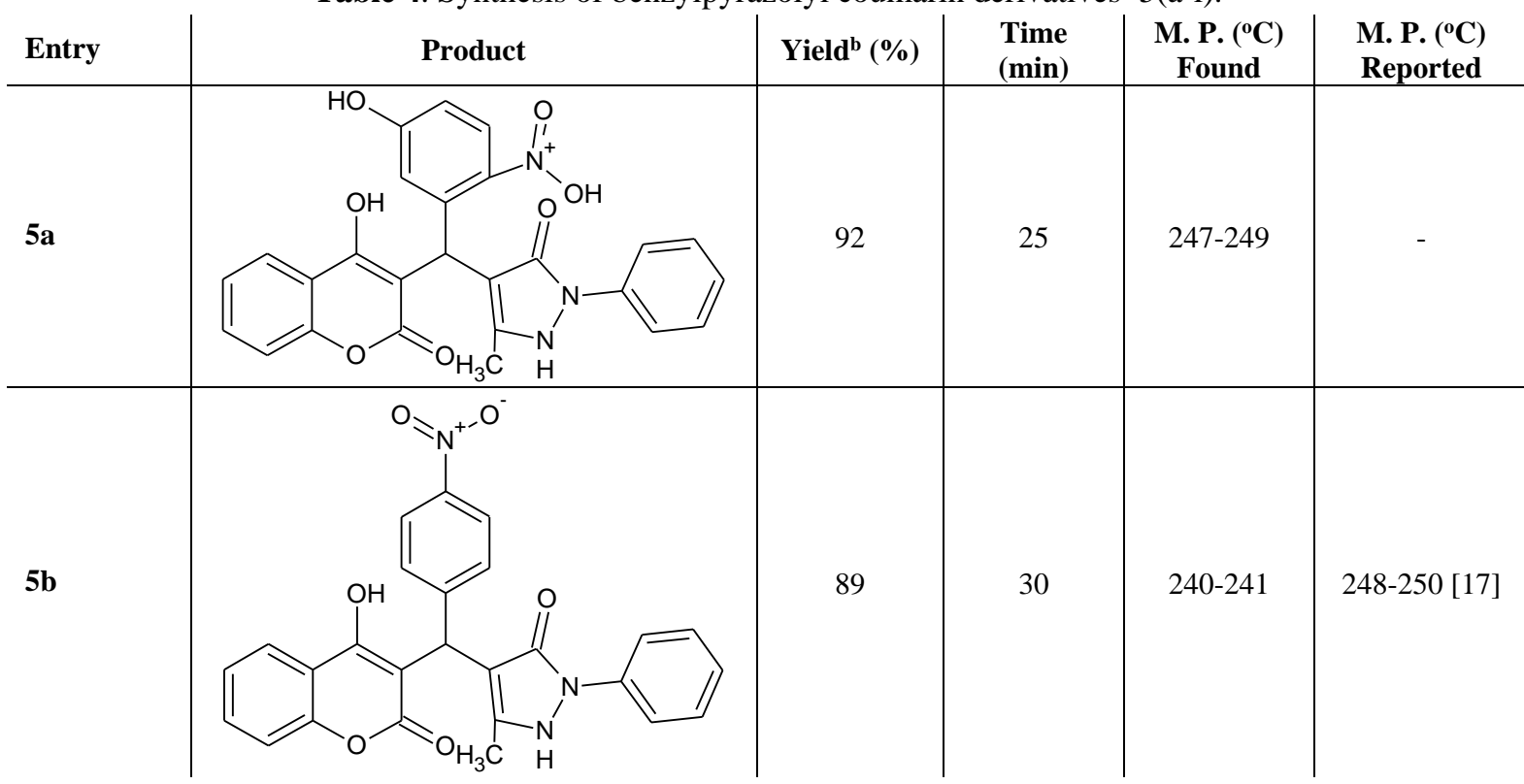


https://doi.org/10.33263/LIANBS103.25252534

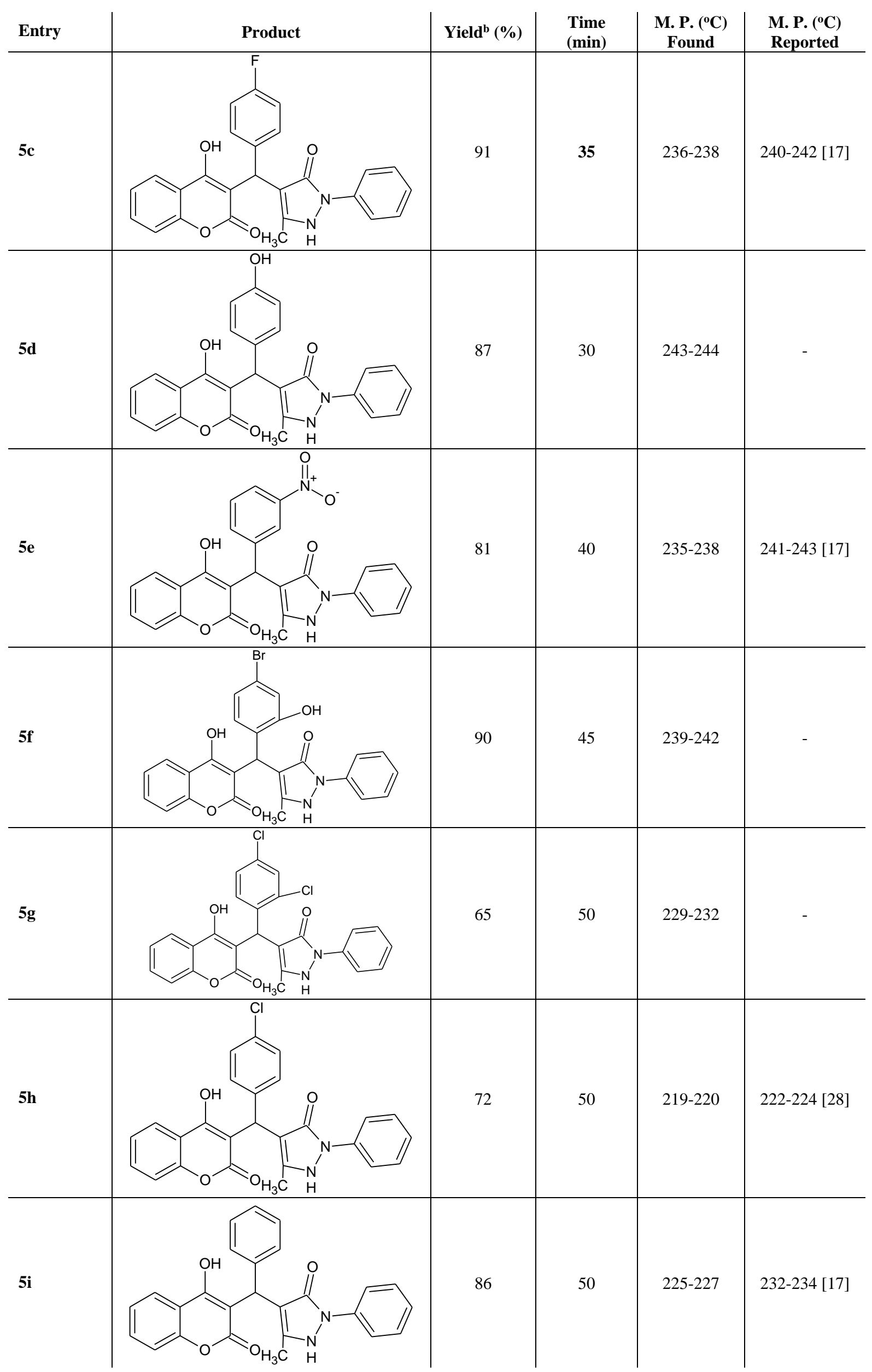

https://nanobioletters.com/

2531 


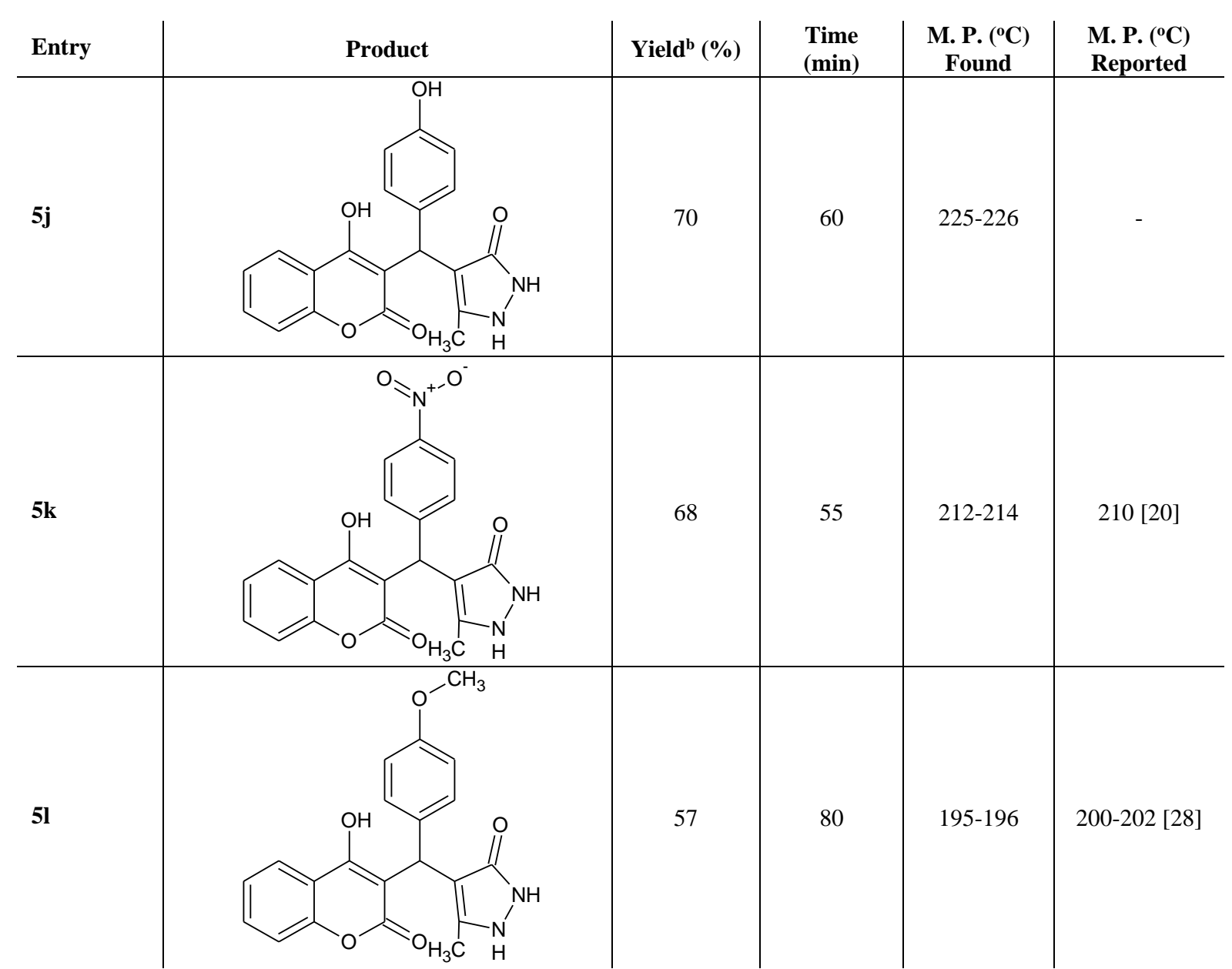

a All reactions were carried out by hydrazine hydrate or phenyl hydrazine $(1 \mathrm{mmol})$, ethyl acetoacetate $(1 \mathrm{mmol})$ aromatic aldehyde $(1 \mathrm{mmol}) \&$ 4-hydroxycoumarin $(1 \mathrm{mmol})$ in $(15 \mathrm{~mL})$ water using $(75 \mathrm{mg})$, Amberlite IR-120 as the catalyst at reflux condition. ${ }^{\mathrm{b}}$ Isolated yield of the product.

The generality of this method was tested by carrying out a condensation reaction with different aldehydes and hydrazines. We found that the methodology is feasible for electrondonating and electron-withdrawing substituents attached to aromatic aldehydes and easily converted into a product without any electronic effect. In the case of hydrazines, phenylhydrazine was smoothly converted into a product within a short time, and high yields were isolated rather than in the case of hydrazine hydrate (Table 4).

\section{Conclusions}

In conclusion, we have demonstrated an alternative protocol for the green and costeffective synthesis of benzylpyrazolyl coumarin via four-component condensation of hydrazine hydrate or phenyl hydrazine, ethyl acetoacetate, aromatic aldehyde, and 4-hydroxy coumarin in an aqueous medium by using Amberlite IR-120 as a catalyst. An aqueous mediated heterogeneous catalyzed multicomponent reaction method addresses the green and environment-friendly method of synthesis.

\section{Funding}

This research received no external funding. 


\section{Acknowledgments}

The authors are thankful to the Principal Deogiri College, Aurangabad, for providing necessary laboratory facilities.

\section{Conflicts of Interest}

The authors declare no conflict of interest.

\section{References}

1. Anastas, P.; Eghbali, N. Green chemistry: Principles and practice. Chem. Soc. Rev. 2010, 39, 301-312, https://doi.org/10.1039/B918763B.

2. Li, C.-J.; Trost, B.M. Green chemistry for chemical synthesis. Proc. Natl. Acad. Sci. 2008, 105, 1319713202, https://doi.org/10.1073/pnas.0804348105.

3. Sunderhaus, J.D.; Martin, S.F. Applications of multicomponent reactions to the synthesis of diverse heterocyclic scaffolds. Chem. Weinh. Bergstr. Ger. 2009, 15, 1300-1308, https://doi.org/10.1002/chem.200802140.

4. Simon, M.-O.; Li, C.-J. Green chemistry oriented organic synthesis in water. Chem. Soc. Rev. 2012, 41, 1415-1427, https://doi.org/10.1039/C1CS15222J.

5. Sheldon, R.A.; Arends, I.; Hanefeld, U. Green Chemistry and Catalysis. John Wiley \& Sons, 2007.

6. Suttle, J.W. Warfarin and Vitamin K. Clin. Cardiol. 1990, 13, VI-16-VI-18, https://doi.org/10.1002/clc.1990.13.s6.16.

7. Hesse, S.; Kirsch, G. A Rapid Access to coumarin derivatives (using Vilsmeier-Haack and Suzuki crosscoupling reactions). Tetrahedron Lett. 2002, 43, 1213-121, https://doi.org/10.1016/S0040-4039(01)023735 .

8. Jung, J.-C.; Lee, J.-H.; Oh, S.; Lee, J.-G.; Park, O.-S. Synthesis and antitumor activity of 4-hydroxycoumarin derivatives. Bioorg. Med. Chem. Lett. 2004, 14, 5527-5531, https://doi.org/10.1016/j.bmcl.2004.09.009.

9. Lee, B.H.; Clothier, M.F.; Dutton, F.E.; Conder, G.A.; Johnson, S.S. Anthelmintic $\beta$-hydroxyketoamides (BKAs). Bioorg Med Chem Lett 1998, 8, 3317-3320, https://doi.org/10.1016/S0960-894X(98)00588-5.

10. Himly, M.; Jahn-Schmid, B.; Pittertschatscher, K.; Bohle, B.; Grubmayr, K.; Ferreira, F.; Ebner, H.; Ebner, C. IgE-mediated immediate-type hypersensitivity to the pyrazolone drug propyphenazone. J. Allergy Clin. Immunol. 2003, 111, 882-888, https://doi.org/10.1067/mai.2003.163.

11. Wu, T.-W.; Zeng, L.-H.; Wu, J.; Fung, K.-P. Myocardial protection of MCI-186 in rabbit ischemiareperfusion. Life Sci. 2002, 71, 2249-2255, https://doi.org/10.1016/S0024-3205(02)01965-3.

12. Sommani, P.; Arai, T.; Yamashita, K.; Miyoshi, T.; Mori, H.; Sasada, M.; Makino, K. Effects of Edaravone on Singlet Oxygen released from activated human neutrophils. J. Pharmacol. Sci. 2007, 103, 117-120, https://doi.org/10.1254/jphs.SC0060170.

13. Xu, Z.; Gao, C.; Ren, Q.-C.; Song, X.-F.; Feng, L.-S.; Lv, Z.-S. Recent advances of pyrazole-containing derivatives as anti-tubercular agents. Eur. J. Med. Chem. 2017, 139, 429-440, https://doi.org/10.1016/j.ejmech.2017.07.059.

14. Mariappan, G.; Saha, B.P.; Sutharson, L.; Singh, A.; Garg, S.; Pandey, L.; Kumar, D. Analgesic, antiinflammatory, antipyretic and toxicological evaluation of some newer 3-methyl pyrazolone derivatives. Saudi Pharm. J. 2011, 19, 115-122, https://doi.org/10.1016/j.jsps.2011.01.003.

15. Bailey, D.M.; Hansen, P.E.; Hlavac, A.G.; Feigensonf, M.E. 3,4-Diphenyl-1H-pyrazole-1-propanamhe antidepressants. Journal of Medicinal Chemistry 1985, 28, 256-260, https://doi.org/10.1021/jm00380a020.

16. Rosiere, C.E.; Grossman, M.I. An Analog of Histamine that Stimulates Gastric Acid Secretion without other Actions of Histamine. Science 1951, 113, 651-651, https://doi.org/10.1126/science.113.2945.651.

17. Ghosh, P.P.; Pal, G.; Paul, S.; Das, A.R. Design and Synthesis of benzylpyrazolyl coumarin derivatives via a four-component reaction in water: investigation of the weak interactions accumulating in the crystal structure of a signified compound. Green Chem. 2012, 14, 2691-2698, https://doi.org/10.1039/C2GC36021G.

18. Karami, B.; Eskandari, K.; Khodabakhshi, S.; Hoseini, S.J.; Hashemian, F. Green synthesis of three substituted methane derivatives by employing $\mathrm{ZnO}$ nanoparticles as a powerful and recyclable catalyst. $R S C$ Adv. 2013, 3, https://doi.org/10.1039/c3ra42993h.

19. Saha, A.; Payra, S.; Banerjee, S. One-pot multicomponent synthesis of highly functionalized bio-active pyrano[2,3-c]pyrazole and benzylpyrazolyl coumarin derivatives using $\mathrm{ZrO}_{2}$ nanoparticles as a reusable catalyst. Green Chem. 2015, 17, 2859-2866, https://doi.org/10.1039/C4GC02420F.

20. Saha, A.; Payra, S.; Verma, S. K.; Mandal, M.; Thareja, S.; Banerjee, S. In silico binding affinity to cyclooxygenase-II and green synthesis of benzylpyrazolyl coumarin derivatives. RSC Adv. 2015, 5, 100978100983, https://doi.org/10.1039/C5RA16643H. 
21. Jadhav, S.; Shioorkar, M.; Chavan, O.s.; Sarkate, A.; Shinde, D.; Pardeshi, R. An alum [KAl (SO 4 ) $2.12 \mathrm{H}$ $2 \mathrm{O}]$ catalyzed microwave assisted multicomponent synthesis of bioactive functionalized benzylpyrazolyl coumarin and quinolinone derivatives in PEG. Chemistry and Materials Research 2015, 7.

22. Yaragorla, S.; Pareek, A.; Dada, R. Ca(II)-Catalyzed, One-pot four component synthesis of functionally embellished benzylpyrazolyl coumarins in water. Tetrahedron Lett. 2015, 56, 4770-4774, https://doi.org/10.1016/j.tetlet.2015.06.049.

23. Eskandari, K.; Karami, B.; Khodabakhshi, S.; Farahi, M. A Highly Efficient tandem Knoevenagel/Michael reaction using Mohr's salt hexahydrate as a green and powerful catalyst: selective synthesis of benzylpyrazolocoumarins on water. J. Chin. Chem. Soc. 2015, 62, 473-478, https://doi.org/10.1002/jccs.201400283.

24. Piruzmand, Z.; Safaei-Ghomi, J.; Ghasemzadeh, M.J.M.C. A facile solvent-free route for the one-pot multicomponent synthesis of benzylpyrazolyl coumarins catalyzed by $\mathrm{FeCl} 3$. $\mathrm{SiO} 2$ nanoparticles. Bulgarian Chemical Communications 2016, 9 ,

25. Valiey, E.; Dekamin, M.G.; Alirezvani, Z. Melamine-modified chitosan materials: An efficient and recyclable bifunctional organocatalyst for green synthesis of densely functionalized bioactive dihydropyrano[2,3-c]pyrazole and benzylpyrazolyl coumarin derivatives. Int. J. Biol. Macromol. 2019, 129, 407-421, https://doi.org/10.1016/j.ijbiomac.2019.01.027.

26. Shaikh, I.R.; Maldar, N.M.N.; Sunyong, C.; Pawar, R.C.; Park, H.-H.; Khwaja-Husain, U. MWCNT incorporated silica aerogel prepared by ambient pressure drying: A recyclable catalyst for multicomponent synthesis of benzylpyrazolyl coumarin at room temperature. Quarterly Journal of Iranian Chemical Communication 2018, 6, 19-29.

27. Mahdizadeh Ghohe, N.; Tayebee, R.; Amini, M.M. Synthesis and characterization of mesoporous $\mathrm{NbZr} / \mathrm{KIT}-6$ as a productive catalyst for the synthesis of benzylpyrazolyl coumarins. Mater. Chem. Phys. 2019, 223, 268-276, https://doi.org/10.1016/j.matchemphys.2018.10.067.

28. Chate, A.V.; Shaikh, B.A.; Bondle, G.M.; Sangle, S.M. Efficient atom-economic one-pot multicomponent synthesis of benzylpyrazolyl coumarins and novel pyrano[2,3-c]pyrazoles catalysed by 2aminoethanesulfonic acid (taurine) as a bio-organic catalyst. Synth. Commun. 2019, 49, 2244-2257, https://doi.org/10.1080/00397911.2019.1619772.

29. Kamal, A.; Babu, K.S.; Hussaini, S.M.A.; Mahesh, R.; Alarifi, A. Amberlite IR-120H, an efficient and recyclable solid phase catalyst for the synthesis of quinoxalines: A greener approach. Tetrahedron Lett. 2015, 56, 2803-2808, https://doi.org/10.1016/j.tetlet.2015.04.046.

30. Kaminwar, N.S.; Tekale, S.U.; Chidrawar, A.B.; Kótai, L.; Pawar, R.P. Eco-friendly synthesis of 1, 4dihydropyrano-[2,3-c] pyrazoles using copper nanoparticles grafted on carbon microsphere as a heterogeneous catalyst. Letters in Applied NanoBioScience 2020, 9, 1521-1528, https://doi.org/10.33263/LIANBS94.15211528.

31. More, Y.W.; Tekale, S.U.; Kaminwar, N.S.; Kótai, L.; Pasinszki, T.; Kendrekar, P.S.; Pawar, R.P. Synthesis of 3, 4-dihydropyrano [c] chromenes using carbon microsphere supported copper nanoparticles $(\mathrm{Cu}-\mathrm{NP} / \mathrm{C})$ prepared from loaded cation exchange resin as a catalyst. Current Organic Synthesis 2019, 16, 288-293, https://doi.org/10.2174/1570179415666181116104931.

32. Tekale, S.U.; Munde, S.B.; Kauthale, S.S.; Pawar, R.P. An efficient, convenient, and solvent-free synthesis of 2,3-dihydroquinazolin-4(1H)-ones using montmorillonite-KSF clay as a heterogeneous catalyst. Organic Preparations and Procedures International 2018, 50, 314-322, https://doi.org/10.1080/00304948.2018.1462058. 